\title{
Onkologie-Pipeline \\ Spezialisierung auf gezielt wirkende Biopharmazeutika
}

Gezielt an den für die Tumorentstehung und das Tumorwachstum spezifischen Mechanismen anzugreifen und die Patientensubgruppe zu selektieren, die am meisten von der gezielten Therapie profitiert, mit diesem Anspruch entwickelt ImClone Systems Biopharmazeutika für die Tumorbehandlung. Vielversprechend sind die Antikörper Ramucirumab und Necitumumab, die bereits in Phase-III-Studien untersucht werden.

ImClone Systems ist eine Tochterfirma von Eli Lilly and Company, die die gesamte Entwicklung und Produktion von Biopharmazeutika leisten kann. Das ist das Besondere an ImClone Systems innerhalb von Lilly Oncology, so Dr. Jerry Kinzel, Branchburg/USA. Besonders sind auch die Kooperationsmöglichkeiten mit den in unmittelbarer Nachbarschaft angesiedelten Instituten, Kliniken und Firmen am Forschungsstandort Manhattan, New York/USA.

Unter dem Motto "Change the World of Cancer Care" werden basierend auf gut belegten wissenschaftlichen Hypothesen Wirkstoffe entwickelt, die die Aussicht haben, First- oder Best-inClass-Substanzen zu werden. Wichtigstes Ziel müsse es sein, so Dr. Greg Plowman, Indiannapolis/USA, überzeugende Evidenz für die Wirkstoffe zu schaffen, damit sich Patienten, Ärzte, Behörden und Kostenträger klar für diese entscheiden können. Dazu bedarf es einer Definition des klinischen Nutzens und nicht allein statistischer Endpunkte. Plowman ist davon überzeugt, dass das wichtigste Ziel nur mit einer personalisierten medizinischen Versorgung der Patienten erreicht werden kann, bei der die Therapiewahl insbesondere auf der Definition und Selektion von Patientengruppen mittels bestimmter Biomarker beruht.

\section{VEGFR-2-Antikörper Ramucirumab}

Ramucirumab gehört zu den Angiogeneseinhibitoren. Der humane Antikörper ist anders als die bislang verfügbaren Angiogeneseinhibitoren spezifisch gegen den Vascular Endothelial Growth Factor Receptor 2 (VEGFR-2) gerichtet. VEGFR-2 ist für Plowman der wichtige Mediator der Tumorangiogenese. Mit der Aktivierung des Rezeptors sind aber auch andere zelluläre Veränderungen verbunden, aus denen Tumorwachstum und Metastasierung resultieren (Abb.). Phase-I- und Phase-II-Daten zur Therapie mit Ramucirumab bei soliden Tumoren deuten darauf hin, dass der VEGFR-2-Antikörper bei intensiv vorbehandelten Patienten wirkt (Spratlin J et al., 2011, Curr Oncol Rep 13: 97-102). Das trifft auch dann zu, wenn die Patienten mit anderen Angiogeneseinhibitoren vortherapiert sind, wie die Ergebnisse einer Phase-II-Studie zur Therapie des metastasierten Nierenzellkarzinoms zeigen (Garcia JA et al., 2010, ASCO - Genitourinary Cancers Symposium: \#326). In diese Studie wurden 40 mit Sunitinib und/ oder Sorafenib vorbehandelte Patienten eingeschlossen. Eine partielle Response oder eine Krankheitsstabilisierung erreichten $74 \%$ der Patienten; das mediane progressionsfreie Überleben betrug 8,3 Monate.

Auch zur First-line-Therapie mit Ramucirumab liegen z.B. aus einer Phase-II-Studie zur Behandlung des fortgeschrittenen nichtkleinzelligen Lungenkarzinoms (NSCLC) vielversprechende Daten vor (Camidge DR et al., 2010, ASCO: \#7588). In der Stu-

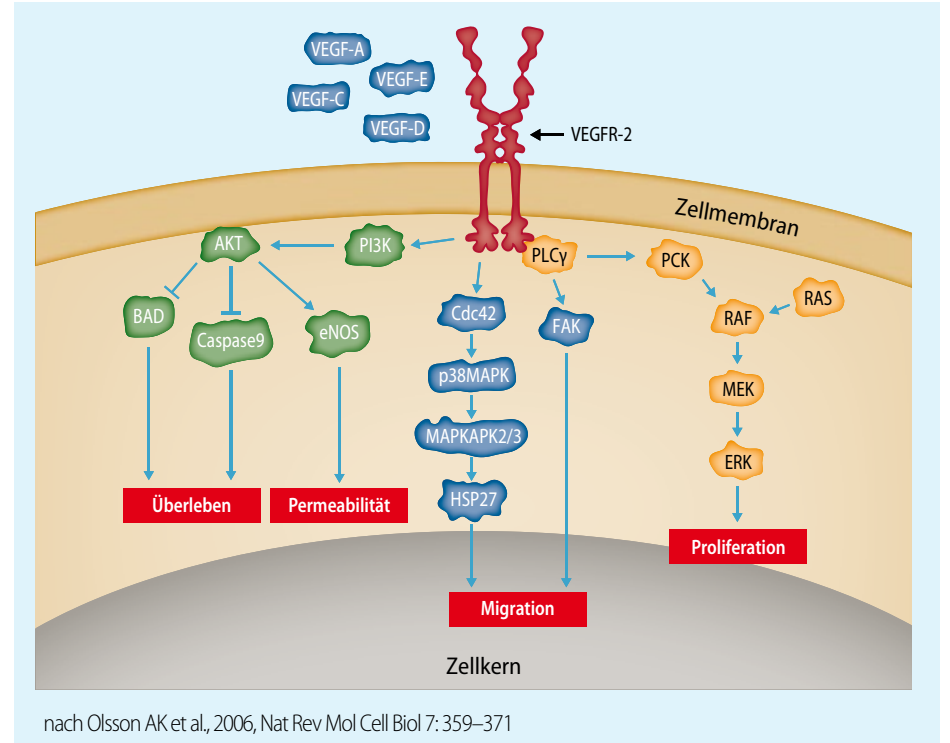

Zelluläre Veränderungen nach Aktivierung des VEGFR-2

die wurden mit Paclitaxel/Carboplatin plus Ramucirumab eine Krankheitskontrollrate von 97\%, eine Ansprechrate von 52\% und ein medianes progressionsfreies Überleben von 5,8 Monaten erreicht. Aktuell wird der VEGFR-2-Antikörper in Phase-III-Studien als Second-line-Therapie bei hepatozellulärem Karzinom, Magenkarzinom, kolorektalem Karzinom und NSCLC sowie als Firstline-Therapie bei Mammakarzinom untersucht.

\section{EGFR-Antikörper Necitumumab}

Necitumumab ist ein gegen den Epidermal Growth Factor Receptor (EGFR) gerichteter humaner Antikörper. Dadurch, aber auch durch weitere Charakteristika, wie z.B. Bindungseigenschaften, unterscheidet sich Necitumumab von dem ebenfalls bei ImClone Systems entwickelten chimären EGFR-Antikörper Cetuximab, so Dr. Bernhard Ehmer, Branchburg/USA. Neben optimierten Wirkstoffcharakteristika ist laut Ehmer aber auch eine optimierte klinische Prüfung hervorzuheben. Basierend auf Erkenntnissen der klinischen Prüfung anderer EGFR-Antikörper werden neue Indikationen, Therapiesituationen und Kombinationen untersucht. Vorrangig werden dabei Indikationen gewählt, für die dringend neue Therapieoptionen benötigt werden.

In einer Phase-III-Studie (SQUIRE) wird Necitumumab plus Gemcitabin/Cisplatin als First-line-Therapie bei fortgeschrittenem plattenepithelialem NSCLC untersucht. $A M$

Quelle: Literatur; Pressereise Lilly Oncology - Eli Lilly and Company, Indiannapolis/USA, ImClone Systems, Manhattan, New York/USA, bzw. Branchburg/USA 